\title{
Transesophageal echocardiography in patients with cryptogenic cerebral ischemia
}

\author{
Fabian Knebel*1, Florian Masuhr ${ }^{2}$, Wolfram von Hausen ${ }^{3}$, Torsten Walde ${ }^{4}$, \\ Henryk Dreger ${ }^{1}$, Vanessa Raab ${ }^{5}$, Mahsun Yuerek ${ }^{6}$, Gert Baumann ${ }^{1}$ and \\ Adrian C Borges ${ }^{1}$
}

\begin{abstract}
Address: ${ }^{1}$ Charité Universitatsmedizin Berlin, Campus Mitte, Medizinische Klinik und Poliklinik mit Schwerpunkt Kardiologie und Angiologie, Berlin, Germany, ${ }^{2}$ Charité Universitatsmedizin Berlin, Campus Mitte, Department of Neurology, Berlin, Germany, ${ }^{3}$ Bundeswehrkrankenhaus Berlin, Abteilung 1, Innere Medizin, Scharnhorststrasse 13, 10115 Berlin, Germany, ${ }^{4}$ Paritätisches Krankenhaus Lichtenberg, Fanningerstraße 32, 10365 Berlin, Germany, ${ }^{5}$ Städtisches Klinikum München Schwabing, Kölner Platz 1, 80804 München, Germany and ${ }^{6}$ State University of New York Downstate Medical Center, Children's Hospital at Downstate 450 Clarkson Avenue, Box 49, 11203 Brooklyn, New York, USA
\end{abstract}

Email: Fabian Knebel* - fabian.knebel@charite.de; Florian Masuhr - florian.mashur@charite.de; Wolfram von Hausen -wgv.hausen@web.de; Torsten Walde - t-walde@gmx.de; Henryk Dreger - henryk.dreger@charite.de; Vanessa Raab - v.witzel@yahoo.de;

Mahsun Yuerek - yuerek@gmx.de; Gert Baumann - gert.baumann@charite.de; Adrian C Borges - adrian.borges@charite.de

* Corresponding author

Published: 28 March 2009

Cardiovascular Ultrasound 2009, 7:15 doi:10.1186/1476-7/20-7-15
Received: 26 February 2009

Accepted: 28 March 2009

This article is available from: http://www.cardiovascularultrasound.com/content/7/I/15

(c) 2009 Knebel et al; licensee BioMed Central Ltd.

This is an Open Access article distributed under the terms of the Creative Commons Attribution License (http://creativecommons.org/licenses/by/2.0), which permits unrestricted use, distribution, and reproduction in any medium, provided the original work is properly cited.

\begin{abstract}
Background: In about one third of all patients with cerebral ischemia, no definite cause can be identified (cryptogenic stroke). In many patients with initially suspected cryptogenic stroke, however, a cardiogenic etiology can eventually be determined. Hence, the aim of this study was to describe the prevalence of abnormal echocardiographic findings in a large number of these patients.

Method: Patients with cryptogenic cerebral ischemia (ischemic stroke, IS, and transient ischemic attack, TIA) were included. The initial work-up included a neurological examination, EEG, cCT, CMRT, 12-lead ECG, Holter-ECG, Doppler ultrasound of the extracranial arteries, and transthoracic echocardiography. A multiplane transeophageal echocardiography (TEE, including i.v. contrast medium application [Echovist], Valsalva maneuver) was performed in all patients

Results: 702 consecutive patients ( 380 male, 383 IS, 319 TIA, age 18-90 years) were included. In $52.6 \%$ of all patients, TEE examination revealed relevant findings. Overall, the most common findings in all patients were: patent foramen ovale (21.7\%), previously undiagnosed valvular disease (15.8\%), aortic plaques, aortic valve sclerosis, atrial septal aneurysms, regional myocardial dyskinesia, dilated left atrium and atrial septal defects. Older patients $(>55$ years, $n=291$ ) and patients with IS had more relevant echocardiographic findings than younger patients or patients with TIA, respectively $(p=0.002, p=0.003)$. The prevalence rates of PFO or ASD were higher in younger patients (PFO: $26.8 \%$ vs. $18.0 \%, p=0.005$, ASD: $9.6 \%$ vs. $4.9 \%, p=0.014$ ).

Conclusion: A TEE examination in cryptogenic stroke reveals contributing cardiogenic factors in about half of all patients. Younger patients had a higher prevalence of PFO, whereas older patients had more frequently atherosclerotic findings. Therefore, TEE examinations seem indicated in all patients with cryptogenic stroke - irrespective of age - because of specific therapeutic consequences.
\end{abstract}




\section{Background}

Cerebral ischemia is among the most common causes of hospitalization, morbidity and mortality in western civilizations. Stroke databases suggest that despite intensive evaluation, approximately $40 \%$ of all patients suffering ischemic strokes have no clearly identifiable cause and $15-20 \%$ of the ischemic events occur in younger patients ( $<55$ years). In the literature, however, there is no consensus on the percentage of ischemic strokes caused by cardioembolic events [1].

Interatrial communications (ASD, PFO with and without atrial septal aneurysms [ASA]) can lead to paradoxical stroke and increase the risk for recurrent thrombembolic cerebral events. [2-4].

In an autopsy study, the overall prevalence of a PFO was 27.3\%. [5] A meta-analysis has shown that a PFO is more common in patients younger than $<55$ years with suspected cryptogenic stroke than in healthy controls [6]. Hence, a PFO is considered as a risk factor for ischemic cerebral events in younger patients. In older patients (i.e. $>55$ years), the association between strokes and prevalence of a PFO is much weaker [7].

In clinical practice, it is not easy to verify paradoxical embolism. In current guidelines, there is no consensus on the clinical relevance of a PFO in cryptogenic stroke and on the indication for the closure of a PFO after a stroke [8].

The aim of this study was to determine the prevalence of echocardiographic findings by TEE in a large number of patients with cryptogenic cerebral ischemia with a focus on pre-defined subgroups (age, sex, stroke vs. TIA).

\section{Methods}

In this single center retrospective study, 702 consecutive patients with acute cerebral ischemia were examined by TEE (1996-2001). All patients underwent neurological clinical examination, electroencephalogram, cCT or cMRT, and laboratory tests. All patients had a 12-leadECG, Holter-ECG, extracranial color-coded sonography, transthoracic echocardiography with no explanations for cerebral ischemia.

Exclusion criteria were: hemodynamically relevant stenoses of the intra- and extracranial arteries, cerebral hemorrhage, atrial fibrillation, migraine, epilepsy, intracranial tumors, acute myocardial infarction in the previous four weeks, previously diagnosed chronic heart failure and left ventricular aneurysms and less than 18 years of age.

Ischemic stroke was defined as a cerebrovascular event with symptoms lasting longer than 24 hours. Diagnosis of ischemic stroke or TIA was confirmed by a study neurologist based on clinical syndrome and results of diagnostic tests, including CT/MRI scans.

Transesophageal echocardiography was performed with a multiplane probe $(5-7 \mathrm{MHz})$ on a Vivid 5 (TEE probe MPTE 5MHz 6A, GE Vingmed, Horton, Norway) and a HP Sonos 5500 (TEE probe HP 21369A; Hewlett-Packard, Paolo Alto, California, USA).

Before transesophageal echocardiography, intraoral xylocaine spray and intravenous midazolam were administered according to the requirements of the patient. The heart rhythm was monitored by ECG during the examination.

Each TEE examination included the standard views and measurement of blood velocities in the left atrial appendage, intravenous administration of contrast agent and Valsalva manoeuvre to exclude interatrial communications (according to [9]).

The images were stored digitally and analyzed off-line by EchoPac PC Dimension (GE Vingmed, Horton Norway) and additionally on video. Echovist 300 was used as contrast medium (Galactose; Schering, Berlin, Germany). Echovist is approved for the diagnosis of intra-atrial communications.

Written consent was obtained from each patient for the TEE, and the ethics committee of the Charite University Hospital approved the protocol.

\section{Statistics}

Statistics were calculated by Statgraphics plus (Version 6, Herndon, Virginia USA). Results are expressed as mean $( \pm$ standard deviation). Comparisons of parametric variables between the responders and the non-responders were calculated by paired Student's t-test. The comparison of echocardiographic parameters between groups was calculated by unpaired Whitney-Mann test. Dichotomized data were analyzed by the $\mathrm{Chi}^{2}$-test. The level of significance was $\mathrm{p} \leq 0.05$.

\section{Results}

702 consecutive patients were included. 380 (54.1\%) were male, 322 (45.9\%) female. 383 (54.6\%) of the patients had an IS and $319(45.4 \%)$ had a TIA. 411 patients were younger than 55 years (58.5\%) and 291 patients were older than 55 years $(41.5 \%)$.

The stroke subgroup was significantly older and had more frequently pathological TEE findings than the patients suffering from a TIA. (Table 1). 
Table I: Patient characteristics (median, \pm SD, $n$ [\%])

\begin{tabular}{|c|c|c|c|c|}
\hline & All $(n=702)$ & Ischemic stroke $(n=383,54.6 \%)$ & TIA $(n=319,45.4 \%))$ & $\mathbf{p}$ \\
\hline Age & $57.1( \pm 15.7)$ & $61.5( \pm 12.3)$ & $51.3( \pm 16.2)$ & $<0.001$ \\
\hline Male Sex (\%) & $380(54.1 \%)$ & $222(57.9 \%)$ & $158(49.5 \%)$ & 0.043 \\
\hline Diabetes mellitus & $13.0 \%$ & $21.2 \%$ & $7.0 \%$ & 0.17 \\
\hline Arterial hypertension & $39.5 \%$ & $59.4 \%$ & $25.6 \%$ & 0.09 \\
\hline Hyperlipidemia & $35.5 \%$ & $50.0 \%$ & $25.6 \%$ & 0.07 \\
\hline Smoker & $26.0 \%$ & $27.6 \%$ & $25.6 \%$ & 0.82 \\
\hline Coronary artery disease & $14.3 \%$ & $21.2 \%$ & $9.3 \%$ & 0.31 \\
\hline Any pathological echo finding & $369(52.6 \%)$ & $225(58.7 \%)$ & $144(45.1 \%)$ & 0.02 \\
\hline Number of pathological findings if any & 2.24 & 2.43 & 1.92 & 0.003 \\
\hline
\end{tabular}

The prevalence of abnormal echocardiographic findings is listed in (Table 2). Neither the presence of a PFO ( $\mathrm{p}=$ $0.053)$, nor of an ASD ( $p=0.65$ ) or an atrial septal aneurysm ( $\mathrm{p}=0.21$ ) was significantly different in the IS and TIA groups. However, findings attributable to atherosclerosis (valvular abnormalities including mitral valve calcification, aortic calcification, aortic valve sclerosis, aortic plaque) were significantly more frequent in the ischemic stroke group (Table 3).

In the older patients ( $>55$ years, $\mathrm{n}=291$, according to $[2,10])$ pathological echocardiographic findings were more frequent $(\mathrm{p}=0.0023)$ than in the younger patients. The prevalence of a PFO was higher in the younger patients (<55 years: $\mathrm{n}=78 ; 26.8 \%,>55$ years: $\mathrm{n}=75$; $18.0 \%$, Pearson's Chi square $\mathrm{p}=0.005)$. The frequency of a ASD was lower in the older patients $(<55$ years: $\mathrm{n}=28$; $9.6 \%,>55$ years: $\mathrm{n}=20 ; 4.9 \%$, Pearson's Chi square $\mathrm{p}=$ 0.014 ); see Figure 1, for examples see Figure 2, Figure 3 and Figure 4 and Additional file 1, Additional file 2 and Additional file 3

The most frequent combination of two findings was PFO+ASA (2.63\% male with IS, $2.71 \%$ male with TIA, $2.63 \%$ female with TIA, $2.71 \%$ female with IS).

\section{Discussion}

This study is to our knowledge the largest TEE study in patients with cryptogenic cerebral ischemia allowing the analysis of the full spectrum of findings and especially the prevalence of a PFO.

In half of all patients, a possible cardiogenic cause was identified by TEE. The most frequent finding was a PFO.
The frequency of positive TEE findings that might explain cerebral ischemia is comparable to previous smaller studies $[11,12]$.

Cardiogenic causes of ischemic stroke can be divided into major (annual incidence of embolic events $>1 \%$ ) and minor risk factors (annual incidence < 1\%) [13]. The major risk factors are atrial fibrillation (1-12\%/year), intracardiac thrombi $(0-35 \%)$, atrial myxoma $(30-40 \%)$, mitral valve stenosis $(8-14 \%$ in sinus rhythm, $31-65 \%$ with concomitant atrial fibrillation), recent myocardial infarction (1-2\%), anticoagulated mechanical heart valves (1.5-3\%), infective endocarditis (12-40\%), dilated cardiomyopathy (4\%) and aortic arch atheromatous plaques (4-16\% especially if $\geq 4 \mathrm{~mm}$ in diameter, [14]). The minor risk factors include mitral valve prolapse $(<0.02 \%)$ and left ventricular aneurysm $(<1 \%)$. Rare causes of cardioembolism include Chiari network, Lambl's excrescences [15], and valvular abnormalities $[16,17]$. Spontaneous echo contrast (SEC) is seen in areas of blood stasis with a slowly moving, cloud-like swirling pattern of "smoke" or increased echogenicity recorded in TEE. However, there is little data on the precise embolic risk of mitral valve annular calcification, spontaneous echo contrast, atrial septal aneurysm, and calcific aortic stenosis [13].

A substantial subset of the cardiogenic factors diagnosed by TEE could have been found with TTE (in combination with trans-cranial Doppler) as well. However, there are no prospective studies showing equivalent diagnostic accuracy of TTE compared to TEE in this setting. Despite the semi-invasivity of TEE, it is still the gold standard for the detection of inter-atrial communications. 
Table 2: Echocardiographic findings in all patients.

\begin{tabular}{lll}
\hline Finding & n & $\%$ \\
\hline Any echocardiographic finding & 369 & 52.6 \\
\hline
\end{tabular}

\begin{tabular}{|c|c|c|}
\hline Patent Foramen ovale (PFO) & 152 & 21.7 \\
\hline Valvular abnormalities & 111 & 15.8 \\
\hline Aortic plaques & 102 & 14.5 \\
\hline Aortic valve sclerosis & 66 & 9.4 \\
\hline Atrial septal aneurysm & 51 & 7.3 \\
\hline regional myocardial dyskinesia in $>2$ segments & 50 & 7.1 \\
\hline Left atrial dilatation & 47 & 6.7 \\
\hline Atrial septal defect & 28 & 4.0 \\
\hline Spontaneous echo contrast (SEC) & 18 & 2.6 \\
\hline Mitral valve prolapse & 15 & 2.1 \\
\hline Valvular vegetations & 14 & 2.0 \\
\hline Aortic valve strands & 14 & 2.0 \\
\hline Intracardial thrombi & 13 & 1.9 \\
\hline mitral valve annulus calcification & 10 & 1.4 \\
\hline Chiari networkt & 6 & 0.9 \\
\hline Aortic valve stenosis & 5 & 0.7 \\
\hline Mitral valve strands & 5 & 0.7 \\
\hline Aortic thrombi & 4 & 0.6 \\
\hline Aortic aneurysm & 3 & 0.4 \\
\hline Prosthetic valve & 3 & 0.43 \\
\hline Left ventricular dilatation & 3 & 0.43 \\
\hline Intracardiac tumor & 1 & 0.14 \\
\hline Mitral valve stenosis & I & 0.14 \\
\hline
\end{tabular}

\section{TIA vs. stroke}

Patients with TIA had less frequently a pathological finding in the TEE. This could be due to the fact that the initially suspected diagnosis "TIA" has a lower specificity with a broader range of other possible non-vascular etiologies such as migraine or focal seizures.

\section{Age}

We found a higher prevalence of PFO and ASD in the younger patients (<55 years). In contrast, Handke [10] reported that PFO are equally distributed in all age groups of patients with cryptogenic stroke. Despite these differences, relevant causes for cerebral ischemia can be identified in all age-groups. Therefore, a restriction of a TEE examination to younger patients with TIA/stroke is not supported by our data.

The current data does not allow a clear recommendation for PFO closure in patients with cryptogenic stroke. Further prospective studies are needed to decide on the clinical advantage of PFO closure, especially in patients $>55$ years of age.

\section{Cardioembolic factors - consequences}

The TEE findings can have specific therapeutic consequences in all age groups (see table 4). Specifically, as the therapeutic consequence of LA-appendage thrombus is oral anticoagulation, its presence should be ruled out before further treatment is limited to platelet aggregation inhibition alone $[13,18]$.

\section{PFO-ASA}

The association of ischemic stroke and PFO is still controversial: A recent large study in a multiethnic population did not confirm an association of PFO and the risk for an ischemic stroke [19]. However, two studies [20,21] have seen a clear association of PFO and ASA in ischemic stroke.

The diagnosis of a PFO or ASA depends on experienced echocardiographers and has - even among experienced examiners - a high inter- and intra-observer variability [3]. Clear diagnostic criteria for intra-atrial defects could reduce the variability [9].

Surgical [22] or percutaneous closure of a PFO is a therapeutic strategy with somewhat inconsistent results $[23,24]$. The percutaneous closure of a PFO after recurrent cerebral ischemia is a safe procedure and leads to a reduction of recurrence rates in the long-term follow-up. Only in a small number of patients, there was a residual shunt with subsequent TIA within the first six months after closure [25]. The recurrent ischemic events in these studies were attributed to a residual post-procedural shunt. Hence, improvement of occluder devices may further reduce the recurrence rates after PFO closure.

Conservative strategies (i.e. antithrombotic therapy) in patients with a PFO are also associated with comparable recurrence rates [26]. However, the risks of antithrom- 
Table 3: The 10 most frequent findings in the stroke and TIA subgroups

\begin{tabular}{|c|c|c|c|}
\hline Rank & Ischemic stroke $(n=383)$ & $\operatorname{TIA}(n=319)$ & P (Chi2 Pearson) \\
\hline 1 & PFO $(94 ; 24.5 \%)$ & PFO (58; $18.2 \%)$ & 0.053 \\
\hline 2 & Valvular abnormalities $(73 ; 19.1 \%)$ & Valvular abnormalities (38; $11.9 \%)$ & 0.014 \\
\hline 3 & Aortic calcification $(73 ; 19.1 \%)$ & Aortic calcification $(32 ; 10.0 \%)$ & 0.001 \\
\hline 4 & Aortic plaque (7I; I8.5\%) & Aortic plaque (31; 9.7\%) & 0.002 \\
\hline 5 & Aoric valve aclerosis (45; $11.7 \%)$ & Aoric valve aclerosis $(21 ; 6.6 \%)$ & 0.029 \\
\hline 6 & Atrial septal aneurysm (36; $9.4 \%)$ & Left atrial dilatation $(20 ; 6.3 \%)$ & LA dilatation: 0.807 \\
\hline 7 & regional myocardial dyskinesia in > 2 segments $(33 ; 8.6 \%)$ & regional myocardial dyskinesia in $>2$ segments $(17 ; 5.3 \%)$ & 0.131 \\
\hline 8 & Left atrial dilatation $(27 ; 7.0 \%)$ & Atrial septal aneurysm (15; 4.7\%) & ASA 0.213 \\
\hline 9 & ASD $(17 ; 4.4 \%)$ & ASD (II; 3.4\%) & 0.65 \\
\hline 10 & $\operatorname{SEC}(16 ; 4.2 \%)$ & Mitral valve prolapse $(6 ; 1.9 \%)$ & \\
\hline
\end{tabular}

botic therapy (platelet inhibition, oral anticoagulation) include bleeding and low compliance rates. A randomized study (PICSS) did not reveal a significant difference in recurrence rates in medically treated patients with or with-

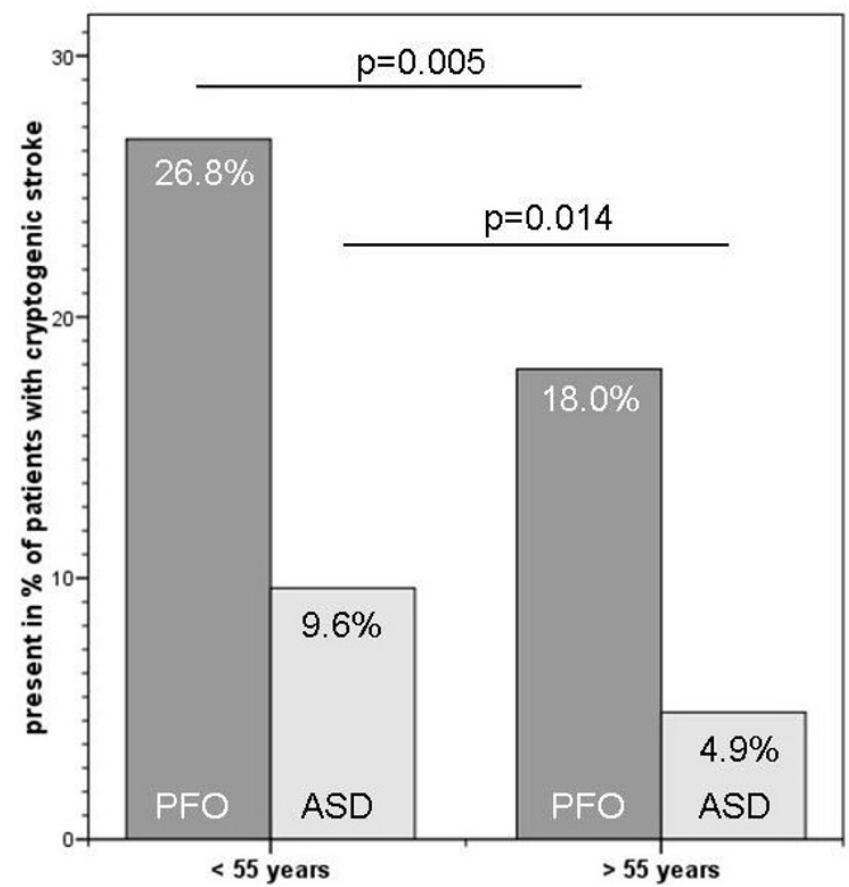

Figure I

Presence of PFO and ASD in the patients with cryptogenic stroke $(n=29 I<55$ years; $n=4 I I>55$ years). out a PFO [4]. Especially in younger patients on medical therapy, the presence of a PFO did not increase the risk of stroke recurrence [27].

In addition to cardiogenic factors, recently, genetic polymorphisms of a variety of genes have been associated with cryptogenic stroke. However, the degree of association and the diagnostic relevance of these genetic polymorphisms is currently not clear [28].

On the background of our study, we recommend the following diagnostic algorithm for patients with cryptogenic

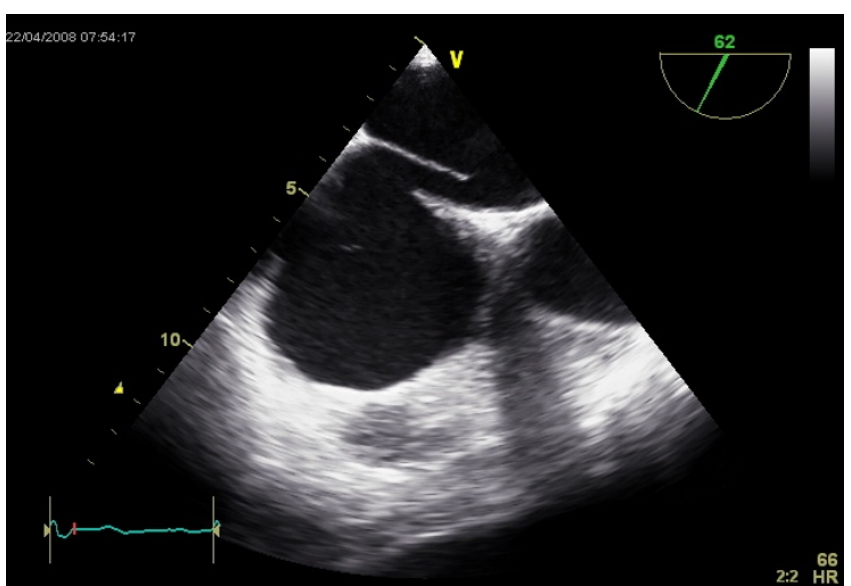

Figure 2

TEE image showing a PFO with the clearly visible interatrial communication. 


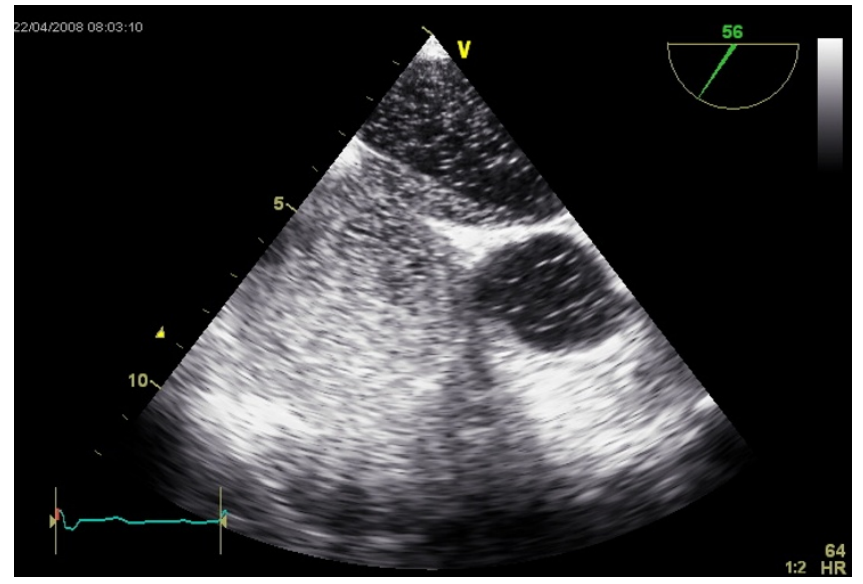

Figure 3

TEE showing the transfer of EchoVist from the right to the left atrium during Valsalva's manoeuvre.

stroke, which as applied in this study: clinical examination, electroencephalogram, cCT or cMRT, 12-lead-ECG, Holter-ECG, extracranial color-coded sonography, transcranial Doppler, transthoracic echocardiography. Only if these examinations do not reveal a cause for the stroke, a TEE seems indicated.

\section{Conclusion}

In conclusion, in this large study in patients with cryptogenic stroke, a TEE reveals in about half of the patients cardiogenic factors that might explain the stroke. Especially, the prevalence of a PFO is higher in the younger

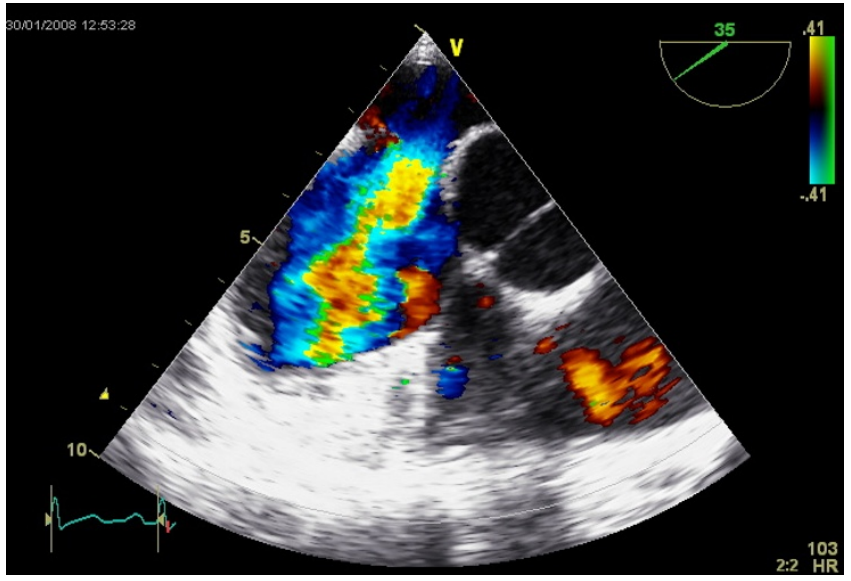

Figure 4

TEE image showing an ASD and the color coded Dopper visualizing the left-to-right shunt.

patients ( $<55$ years of age). Therefore, a TEE seems indicated in all patients with cryptogenic stroke - irrespective of age - because of specific therapeutic consequences.

\section{Limitations}

This study was observational. The quality of observational studies seems to be comparable to randomized controlled trials $[29,30]$. There was no follow-up of the patients concerning recurrence of cerebral ischemia or mortality.

We did not analyze the prevalence of abnormal TEE findings in single-vessel and lacunar strokes in comparison to

Table 4: TEE findings in patients with ischemic stroke and their possible therapeutic consequences.

\begin{tabular}{|c|c|}
\hline TEE finding & Possible therapeutic consequence \\
\hline PFO and ASD & ASS, anticoagulation, operative or interventional device closure \\
\hline aortic plaques & ASS, statin therapy \\
\hline reduced LVEF & oral anticoagulation, ASS, coronary angiography, heart failure therapy \\
\hline left atrial dilatation & further cardiological work-up \\
\hline Spontaneous echo contrast & search for intermittent atrial fibrillation, oral anticoagulation \\
\hline left atrial thrombus & anticoagulation, operation \\
\hline thoracic aneurysm of the aorta & echo control, operation \\
\hline mitral valve prolapse & antiarrhythmic therapy, echo follow-up, anticoagulation \\
\hline aortic/mitral valve stenosis & timing of valve replacement \\
\hline LA-appendage thrombus & oral anticoagulation \\
\hline
\end{tabular}


multi-vessel strokes. The data of our study is historical (1996-2001). Currently, the indication for a TEE in cryptogenic stroke is more selective.

\section{Competing interests}

The authors declare that they have no competing interests.

\section{Author's contributions}

FK and FM equally contributed to the study. FK, FM, WVH, ACB have designed the study, and have performed the examinations. FK, ACB, FM analyzed the data and have written the manuscript. MY, VR, TW, HD performed the TEE examination, collected and interpreted the acquired data. GB has supervised the study and contributed by revising the manuscript critically.

\section{Additional material}

\section{Additional file 1}

Video 1

TEE showing a PFO in $62^{\circ}$

Click here for file

[http://www.biomedcentral.com/content/supplementary/14767120-7-15-S1.avi]

\section{Additional file 2}

Video 2

TEE showing inter-atrial transfer of EchoVist from the right atrium to the left atrium during Valsalva maneuver.

Click here for file

[http://www.biomedcentral.com/content/supplementary/1476-

7120-7-15-S2.avi]

\section{Additional file 3}

\section{Video 3}

TEE showing a large ASD

Click here for file

[http://www.biomedcentral.com/content/supplementary/14767120-7-15-S3.avi]

\section{References}

I. Amarenco P: Cryptogenic stroke, aortic arch atheroma, patent foramen ovale, and the risk of stroke. Cerebrovasc Dis 2005, 20(Suppl 2):68-74.

2. Mas JL, Arquizan C, Lamy C, Zuber M, Cabanes L, Derumeaux G, Coste J: Patent Foramen Ovale and Atrial Septal Aneurysm Study Group. Recurrent cerebrovascular events associated with patent foramen ovale, atrial septal aneurysm, or both. N Engl I Med 200I, 345(24): 1740-6.

3. Cabanes L, Coste J, Derumeaux G, Jeanrenaud X, Lamy C, Zuber M, Mas JL: Patent Foramen Ovale and Atrial Septal Aneurysm Study Group. Interobserver and intraobserver variability in detection of patent foramen ovale and atrial septal aneurysm with transesophageal echocardiography. J Am Soc Echocardiogr 2002, I 5(5):44I-6.

4. Homma S, Sacco RL, Di Tullio MR, Sciacca RR, Mohr JP: PFO in Cryptogenic Stroke Study (PICSS) Investigators. Effect of medical treatment in stroke patients with patent foramen ovale: patent foramen ovale in Cryptogenic Stroke Study. Circulation 2002, 105(22):2625-31.
5. Hagen PT, Scholz DG, Edwards WD: Incidence and size of patent foramen ovale during the first 10 decades of life: an autopsy study of 965 normal hearts. Mayo Clin Proc 1984, 59:17-20.

6. Lechat P, Mas JL, Lascault G, Loron P, Theard M, Klimczac M, Drobinski G, Thomas D, Grosgogeat Y: Prevalence of patent foramen ovale in patients with stroke. $N$ Engl J Med 1988, 3 I 8(18): I | 48-I I52.

7. Overell JR, Bone I, Lees KR: Interatrial septal abnormalities and stroke: a meta-analysis of case-control studies. Neurology 2000, 55(8): I I72-1 I79.

8. Sacco RL, Adams R, Albers G, Alberts MJ, Benavente O, Furie K, Goldstein LB, Gorelick P, Halperin J, Harbaugh R, Johnston SC, Katzan I, Kelly-Hayes M, Kenton EJ, Marks M, Schwamm LH, Tomsick T, American Heart Association; American Stroke Association Council on Stroke; Council on Cardiovascular Radiology and Intervention; American Academy of Neurology: Guidelines for prevention of stroke in patients with ischemic stroke or transient ischemic attack: a statement for healthcare professionals from the American Heart Association/American Stroke Association Council on Stroke: co-sponsored by the Council on Cardiovascular Radiology and Intervention: the American Academy of Neurology affirms the value of this guideline. Stroke 2006, 37(2):577-617.

9. Attaran RR, Ata I, Kudithipudi V, Foster L, Sorrell VL: Protocol for optimal detection and exclusion of a patent foramen ovale using transthoracic echocardiography with agitated saline microbubbles. Echocardiography 2006, 23(7):616-622.

10. Handke M, Harloff A, Olschewski M, Hetzel A, Geibel A: Patent foramen ovale and cryptogenic stroke in older patients. $\mathrm{N}$ Engl J Med 2007, 357(22):2262-2268.

II. Ulrich JN, Hesse B, Schuele S, Vlassak I, Sila CA, Jaber WA: Singlevessel versus multivessel territory acute ischemic stroke: value of transesophageal echocardiography in the differentiation of embolic stroke. J Am Soc Echocardiogr 2006, 19(9): II65-1169.

12. de Bruijn SF, Agema WR, Lammers GJ, Wall EE van der, Wolterbeek R, Holman ER, Bollen EL, Bax JJ: Transesophageal echocardiography is superior to transthoracic echocardiography in management of patients of any age with transient ischemic attack or stroke. Stroke 2006, 37( 10):253I-2534.

13. Kapral MK, Silver FL: Preventive health care, 1999 update: 2. Echocardiography for the detection of a cardiac source of embolus in patients with stroke. Canadian Task Force on Preventive Health Care. CMAJ 1999, 161 (8):989-996.

14. Amarenco P, Duyckaerts C, Tzourio C, Henin D, Bousser MG, Hauw $\mathrm{JJ}$ : The prevalence of ulcerated plaques in the aortic arch in patients with stroke. N Engl J Med I992, 326(4):22 I-225.

15. Siles Rubio JR, Ruiz de Castroviejo del Campo J, Tirado Miranda R, Jansen Chaparro S, Pavlovic D: [Transient ischemic attack due to Lambl's excrescence. Report of a case and review of the literature]. An Med Interna 2006, 23(4): $181-183$.

16. Freedberg RS, Goodkin GM, Perez JL, Tunick PA, Kronzon I: Valve strands are strongly associated with systemic embolization: a transesophageal echocardiographic study. J Am Coll Cardiol 1995, 26(7): $1709-1712$.

17. Tice FD, Slivka AP, Walz ET, Orsinelli DA, Pearson AC: Mitral valve strands in patients with focal cerebral ischemia. Stroke 1996, 27(7): $1|83-1| 86$.

18. Agmon Y, Khandheria BK, Gentile F, Seward JB: Clinical and echocardiographic characteristics of patients with left atrial thrombus and sinus rhythm: experience in 20643 consecutive transesophageal echocardiographic examinations. Circulation 2002,
I05(I):27-3I.

19. Di Tullio MR, Sacco RL, Sciacca RR, jin Z, Homma S: Patent foramen ovale and the risk of ischemic stroke in a multiethnic population. J Am Coll Cardiol 2007, 49(7):797-802.

20. Bonati LH, Kessel-Schaefer A, Linka AZ, Buser P, Wetzel SG, Radue EW, Lyrer PA, Engelter ST: Diffusion-weighted imaging in stroke attributable to patent foramen ovale: significance of concomitant atrial septum aneurysm. Stroke 2006, 37(8):2030-2034.

21. Force M, Massabuau P, Larrue V: Prevalence of atrial septal abnormalities in older patients with cryptogenic ischemic stroke or transient ischemic attack. Clin Neurol Neurosurg 2008, I I 0(8):779-783. 
22. Dearani JA, Ugurlu BS, Danielson GK, Daly RC, McGregor CG, Mullany CJ, Puga FJ, Orszulak TA, Anderson BJ, Brown RD Jr, Schaff HV: Surgical patent foramen ovale closure for prevention of paradoxical embolism-related cerebrovascular ischemic events. Circulation 1999, 100(19 Suppl):III7I-75.

23. Windecker S, Wahl A, Chatterjee T, Garachemani A, Eberli FR, Seiler C, Meier B: Percutaneous closure of patent foramen ovale in patients with paradoxical embolism: long-term risk of recurrent thromboembolic events. Circulation 2000, I0I(8):893-898.

24. Braun M, Gliech V, Boscheri A, Schoen S, Gahn G, Reichmann H, Haass M, Schraeder R, Strasser RH: Transcatheter closure of patent foramen ovale (PFO) in patients with paradoxical embolism. Periprocedural safety and mid-term follow-up results of three different device occluder systems. Eur Heart J 2004, 25(5):424-430.

25. Windecker $S$, Meier $B$ : Is closure recommended for patent foramen ovale and cryptogenic stroke? Patent foramen ovale and cryptogenic stroke: to close or not to close? Closure: what else! Circulation 2008, II 8(19): 1989-1998.

26. Bogousslavsky J, Garazi S, Jeanrenaud X, Aebischer N, Van Melle G: Stroke recurrence in patients with patent foramen ovale: the Lausanne Study. Lausanne Stroke with Paradoxal Embolism Study Group. Neurology 1996, 46(5): I 30I-1305.

27. Homma S, DiTullio MR, Sacco RL, Sciacca RR, Mohr JP, PICSS Investigators: Age as a determinant of adverse events in medically treated cryptogenic stroke patients with patent foramen ovale. Stroke 2004, 35(9):2। 45-2। 49.

28. Bersano A, Ballabio E, Bresolin N, Candelise L: Genetic polymorphisms for the study of multifactorial stroke. Hum Mutat 2008 , 29(6):776-795.

29. Benson K, Hartz AJ: A comparison of observational studies and randomized, controlled trials. N Engl J Med 2000, 342(25): $1878-1886$

30. Concato J, Shah N, Horwitz RI: Randomized, controlled trials, observational studies, and the hierarchy of research designs. $N$ Engl J Med 2000, 342(25): I887-I892.

Publish with Bio Med Central and every scientist can read your work free of charge

"BioMed Central will be the most significant development for disseminating the results of biomedical research in our lifetime. "

Sir Paul Nurse, Cancer Research UK

Your research papers will be:

- available free of charge to the entire biomedical community

- peer reviewed and published immediately upon acceptance

- cited in PubMed and archived on PubMed Central

- yours - you keep the copyright
BioMedcentral 